\title{
Ionic current through an open channel: a low-dimensional model of coupling with vibrations of the wall.
}

\author{
R. Tindjong ${ }^{a}$, A. Applegate ${ }^{a}$, R.S. Eisenberg ${ }^{b}$, I. Kaufman $^{c}$, \\ D. G. Luchinsky ${ }^{a}$, P.V.E. McClintock ${ }^{a}$; \\ ${ }^{a}$ Department of Physics, Lancaster University, Lancaster LA1 4YB, UK; \\ ${ }^{b}$ Department of Molecular Biophysics and Physiology, Rush Medical College, \\ 1750 West Harrison, Chicago, IL 60612, USA; \\ ${ }^{c}$ The Russian Research Institute for Metrological Service, \\ Gosstandart, Moscow, 119361, Russia
}

\begin{abstract}
Ionic motion through an open ion channel is analyzed within the framework of self-consistent Brownian dynamics formalism. A novel conceptual model of coupling of the ion's motion to the vibrations of the pore walls is introduced. The model allows one to include into simulations an important additional mechanism of energy dissipation and the effects of self-induced strong modulation of the channel conductivity.
\end{abstract}

Keywords: ionic channels, Poisson equation, Langevin equation, self-consistent approach

\section{INTRODUCTION}

Research activities at the frontiers of nano-electronics and of biotechnology share not only the common goal of manipulating nanostructures on the scale of individual atoms, but also common physical principles underlying the corresponding computational methods. In particular, the conductance of ionic channels (which are natural nanotubes in cellular membranes that control a vast range of biological functions ${ }^{1}$ ) can be successfully investigated using ideas and techniques developed earlier for the analysis of semiconductor devices. ${ }^{2}$ Moreover it was shown recently that the interaction between exposed gate MOSFET transistors and nerve cells is possible by doping a membrane with ionic channels ${ }^{3}$ this open the prospect of integrating the central nervous system with computer chips. However, understanding the basic physical principles underlying the operation of nano-machinery is still in its infancy due to the extraordinary complexity of the problem.

In the context of ionic channels the challenge is to predict the function - the current of ions of different types and concentrations through a variety of channels - from the structure, given fundamental physical laws. We note that many drugs in present use act directly or indirectly on biological ion channels. Thus the solution of this challenging scientific problem might have significant impact on applied research in the pharmaceutical industry. A number of theoretical and computational methods ranging from continuous grand canonical $(\mathrm{GC})^{4}$ and Poisson-Nernst-Planck (PNP) ${ }^{2}$ theories to the brownian dynamics (BD),$^{5}$ and molecular dynamics $(\mathrm{MD})^{6-8}$ simulations have been successfully applied to gain understanding of various properties of ionic channels on different scales in space and time and with different level of details. The success of the GC and PNP theories suggests that a few basic physical principles may provide deep physical insight into the selectivity and conductivity of ionic channels. However, many phenomena, usually associated with single filing of ions, are usually missed in continuous theories. The available kinetic theories, namely the full-scale 3D BD and MD, are computationally very time consuming and cannot provide a quantitative theory of ions moving through a channel. Therefore, there is a need to develop a self-consistent approach for the description of the directional and ionic selectivity and conductivity of the ionic channels, using basic physical principles. It is important that such an approach is capable of distinguishing contributions to the channel properties of single ions interactions. One

Further author information: (Send correspondence to R.T.)

R.T.: E-mail: r.tindjong@lancaster.ac.uk, Telephone: +44 (0)1524 593079, Address: Department of Physics, Lancaster University, Lancaster LA1 4YB, UK 
of the possibilities here is to analyze in a self-consistent way coupled Langevin Poisson equations as suggested in. ${ }^{9}$

A specific advantage of using the self-consistent Poisson-Langevin approach is the possibility of including new strongly non-equilibrium features in modelling the ionic current through the channel, such as the interaction with the vibrations of the walls. The importance of mechanical interaction with the channel walls follows from the statistical mechanical analysis of pore selectivity ${ }^{4}$ and they can have a dramatic effect on the transition rate in biomolecules. ${ }^{10-12}$

The present paper is a report on the work in progress. We introduce a new simplified model of the ion-wall interaction that allows us to include coupling of the ion's motion to vibrational modes of the pore walls and to consider the effect of such coupling on the transition rates and on dissipation in the channel. This is done within the framework of the self-consistent model of ionic current based on solution of the coupled Langevin and Poisson equations.

The paper is organized as follows. In Sections 2 and 3 we review earlier approximations adopted in lowdimensional self-consistent models of ionic transport in open channels. In the Sections 4 and 5 we suggest a number of improvements that allow us to incorporate into the model the reaction field at the entrance of the channel and, in particular, to introduce a new simple model of coupling of the ionic transport to fluctuations of the channel walls.

\section{ONE DIMENSIONAL APPROXIMATION}

The starting point of our research is the original system of the Langevin equation for a particle of mass $m$ and charge $Z e$ coupled to the Poisson equation ${ }^{13,14}$ suggested in ${ }^{14,15}$ (cf. $^{5,16,17}$ )

$$
\begin{gathered}
m \frac{d^{2} \widetilde{x}}{d \widetilde{t^{2}}}+m \widetilde{\gamma} \frac{d \widetilde{x}}{d \widetilde{t}}+Z e \frac{d \phi}{d \widetilde{x}}=\sqrt{2 \widetilde{\gamma} m k_{b} T} \xi(\widetilde{t}) \\
-\varepsilon \frac{d^{2} \phi}{d \widetilde{x}^{2}}=q P(\widetilde{x})+Z e C(\widetilde{x})+\widetilde{\varepsilon}\left(\widetilde{\Delta}\left(1-\frac{\widetilde{x}}{d}\right)-\phi\right) \\
\phi(0)=0, \quad \phi(d)=V_{a p p}
\end{gathered}
$$

Here $x$ describes the position of the particle, $\phi$ is the electrical potential, $d$ is the length of the channel, $\widetilde{\gamma}$ is the viscous friction coefficient, and $\xi(\widetilde{t})$ is Gaussian zero-mean uncorrelated noise. In equation (2), the first term on the left hand side is the permanent charge per unit volume embedded on the membrane wall, the second term is the charge per unit volume carried by the particle crossing the channel, and the last term is the induced charge. The parameter $\widetilde{\varepsilon} \equiv \frac{\epsilon_{p}}{\epsilon_{H_{2} O}} \cdot \frac{2 \epsilon_{0}}{R^{2} \ln (d / R)}$ and $\widetilde{\Delta}$ is equal to the applied voltage $\left(V_{a p p}\right)$. The permanent charge distribution is one of the main fitting parameters of the model. Equations $(1,2,3)$ are scaled together, using the following dimensionless variables:

$$
x=\frac{\widetilde{x}}{d} ; \quad \Phi=\frac{\phi}{U_{T}} ; \quad t=\frac{\widetilde{t}}{d / \sqrt{k_{B} T / m}} ; \quad \xi=\frac{\widetilde{\xi}}{\sqrt{d \sqrt{m / k_{B} T}}} ; \quad U_{T}=\frac{k_{B} T}{Z e} ; \quad \Delta=\frac{\widetilde{\Delta}}{U_{T}}
$$

$P(\widetilde{x})$ and $C(\widetilde{x})$ are scaled by the inverse of the volume of the channel $v_{0}$. The coupled dimensionless PoissonLangevin equations are then given by:

$$
\begin{gathered}
\frac{d^{2} x}{d t^{2}}+\gamma \frac{d x}{d t}+\frac{d \Phi}{d x}=\sqrt{2 \gamma} \xi(t) \\
\frac{d^{2} \Phi}{d x^{2}}=-\alpha P(x)-\alpha \delta\left(x-x_{0}\right)-\beta^{2}(\Delta(1-x)-\Phi)
\end{gathered}
$$




$$
\Phi(0)=0, \quad \Phi(1)=\Delta
$$

For sake of simplicity, we set $q=e$ in the scaling. From the scaling, the following dimensionless parameters are obtained: $\gamma=d \sqrt{\frac{m}{k}{ }_{B} T} \widetilde{\gamma}$, in which $\widetilde{\gamma}=\frac{k_{B} T}{m D}$ is the friction coefficient and $D$ is the diffusion coefficient of the ion. $\beta^{2}=\frac{d^{2} \widetilde{\varepsilon}}{\epsilon}$, with $\epsilon=\epsilon_{0} \epsilon_{H_{2} O} . \alpha=\kappa^{2} d$ where $\kappa^{-1}=\sqrt{\frac{\epsilon v_{0} U_{T}}{e}}$ can be identified as the Debye length in the channel.

The advantage of the original system of equations $(1,2,3)$ is twofold. On one hand the Poisson equation allows analytical solution. On the other hand it was shown earlier that the corresponding PNP theory can provide good agreement with many experimental results. ${ }^{2}$ The solution of equation (6) for one moving charge located at $x_{0}$ is given by:

$$
\Phi(x)=\frac{\Delta}{\operatorname{sh}(\beta)}(\operatorname{sh}(\beta x)+\operatorname{sh}(\beta(x-1)))-\alpha \int_{0}^{1} G(x, s) P(s) d s-\alpha G\left(x, x_{0}\right),
$$

where

$$
G(x, s)=\frac{1}{\beta \operatorname{sh}(\beta)} \begin{cases}\operatorname{sh}(\beta x) \operatorname{sh}(\beta(s-1)), & 0 \leq x \leq s \\ \operatorname{sh}(\beta s) \operatorname{sh}(\beta(x-1)), & s \leq x \leq 1\end{cases}
$$

However, the form (2) does not allow for the analysis of the reaction field, nor for the shape of the channel at the entrance of the pore.

\section{BOUNDARY CONDITIONS}

Boundary conditions are traditionally a controversial issue in this type of simulation. ${ }^{15,18,19}$ It may be stated unambiguously that the electric potential and the concentration are constant far from the pore mouth. However, close to the entrance of the pore the boundary conditions are formed in a self-consistent way according to the net flux of ions through the pore. In this work we will adopt a simplified version of the boundary conditions that have proved to be effective in reproducing a number of important characteristics of the channels in the framework of the PNP approach. ${ }^{20}$ One of the main goals of the further research, However, will be to verify the consistency of these boundary conditions.

A simplified version of the boundary conditions corresponds to a steady flux towards the hemispherical sink capturing ions at one mouth of the pore (see e.g. ${ }^{1}$ )

$$
\tau_{\text {arrival }}=\frac{1}{2 \pi c D r}
$$

where $c$ is the concentration of particles in the solution, $D$ is the diffusion coefficient, and $r$ is the radius of the pore. Accordingly, we simulate this boundary condition using the Poisson process with an exponential distribution of waiting times between the successive entrances of the ion into the hemispherical sink at the mouth of the pore. We emphasize that the probability for two ions to be present in the hemisphere of radius $r=4 \stackrel{\AA}{ }$ can be neglected (see the distribution of e.g. $K^{+}-K^{+}$ion pairs in the solution found ${ }^{19}$ using BD ).

In the one dimensional approximation it corresponds to the random appearance of an ion both on the left and right hand side of the channel. The position of the ion is uniformly distributed at the segment of length $r$ with velocity distribution of the $u_{x}$ component taken from the Maxwellian distribution. To include the steady flux of the ions towards the pore, the mean value of the velocity is shifted by the magnitude corresponding to the flux (9) (cf. Eisenberg et $\mathrm{al}^{15}$ ).

The potential corresponding to (8) and an example of a trajectory crossing the channel are shown in the Fig. 1. Finally, the total flux can be estimated as ${ }^{15,21}$

$$
J=\frac{1}{d}\left(c_{L} \operatorname{Prob}\{R \mid L\}-c_{R} \operatorname{Prob}\{L \mid R\}\right),
$$




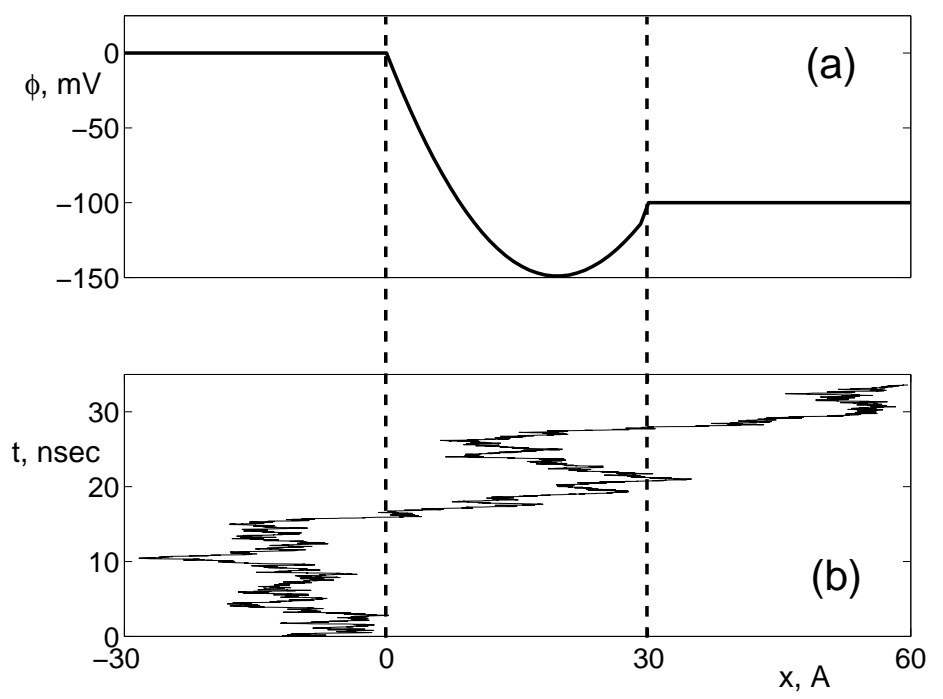

Figure 1. (a) Electric potential in empty pore calculated using (8) with negative fixed charge 1e distributed along the wall according to Gaussian distribution. (b) Ion trajectory crossing the channel starting at the left at time $t=0$, and finishing at the right at $t=35$ nsec.

where $d$ is the channel length and the probabilities of crossing channel from the left to the right $\operatorname{Prob}\{R \mid L\}$ and from the right to the left $\operatorname{Prob}\{L \mid R\}$ have to be found as the result of $\mathrm{BD}$ simulations.

We emphasize that the results of the BD simulations and of the solution of the PNP equations must coincide unless there is some inconsistency in the specification of the boundary conditions. The important difference between the two approaches, however, is the fact that $\mathrm{BD}$ simulations take into account the finite diameters of the ions. Therefore one goal of further research will be to verify the discrepancy between the results of two approaches reported recently by Moy et $\mathrm{al}^{16}$ and Corry et al. ${ }^{17}$ To make the comparison more feasible, and also to be able to include within the framework of the same low-dimensional formalism new physical features of the ion-wall interactions, we suggest in the present work a number of modifications of the one-dimensional approximation outlined above.

\section{TWO DIMENSIONAL POISSON EQUATION}

A more realistic approximation for the electrical force driving the ion near channel axis can be derived by numerical solution of the Poisson equation in three-dimensional space with cylindrical symmetry. In our work we use the Finite Volume (FV) method ${ }^{23}$ with mixed boundary conditions. In this approach one introduces finite volume elements, integrates the Poisson equation over the volume of the corresponding element $V$, and transforms the first volume integral into a surface integral using Gauss's theorem, yielding ${ }^{16}$

$$
\begin{array}{r}
\int_{V}(\nabla(\epsilon \nabla \phi)) d v=-\frac{1}{\epsilon_{0}} \int_{V} \rho(v) d v, \\
\int_{\partial V} \vec{D} \overrightarrow{d s}=\frac{1}{\epsilon_{0}} \int_{V} \rho(v) d v,
\end{array}
$$

where $\vec{D}$ is the electric displacement, $\partial V$ is the boundary of the volume and $\rho(v)$ is the charge density. The schematic illustration of the channel and one of the finite volume elements is shown in the Fig. 2(a) Further details, examples, and free downloads of the $\operatorname{code}^{24}$ are available. It is clear that, to preserve cylindrical symmetry, the ion has to move only along the axis of symmetry, and the fixed charge in the wall must be uniformly distributed in the finite volumes, like that shown in the figure. However, it is a reasonable approximation as long as one assumes continuous dielectric constants of the pore and of the solution and the actual atomic structure 


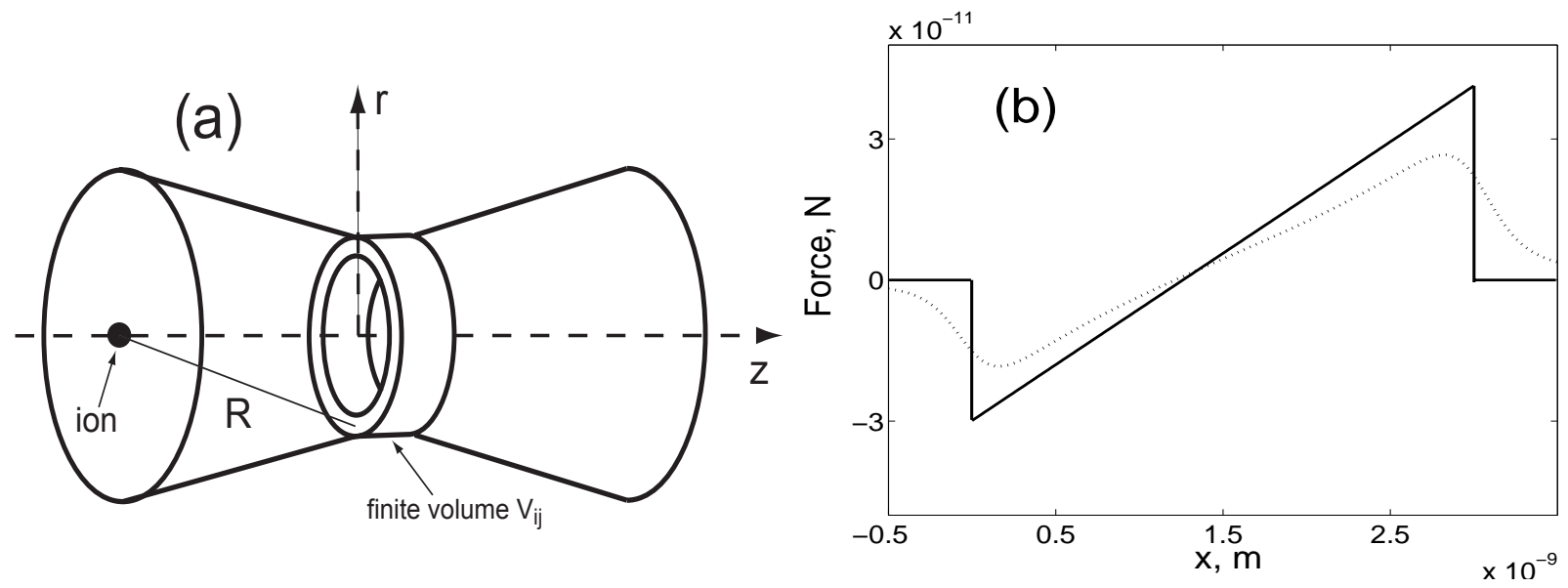

Figure 2. (a) Geometry of the pore with cylindrical symmetry. Example of one of the finite volume elements with width in $z$-direction $h_{z}$ and thickness in $r$-direction $h_{r}$. Ion moves along the axis of symmetry. Fixed charge on the channel wall is uniformly distributed in the finite volume element. $R$ is the distance from ion to the flexible ring discussed in the text. (b) A comparison between forces exerted on the ion in the channel calculated using one-dimensional approximation (8) (solid line) and numerical solution of the poisson equation in cylindrical symmetry (dashed line). The cylindrical pore of radius $r=4 \AA$ and length $d=30 \AA$ is situated between $x=0$ and $x=30 \AA$. The potential is set to be 0 on the left and $-100 \mathrm{mV}$ on the right hand side of the pore in $1 \mathrm{~d}$ approximation and 0 and $-100 \mathrm{mV}$ at some distance from the pore in the case of cylindrical symmetry.

of the pore is not taken into account. In particular, the distribution of the ionic position within the pore is assumed to be sharply peaked on the axis of symmetry.

An important advantages of using this approximation is that one may use low-dimensional simulations to take into account more realistic shapes of the channel (including its spatial asymmetry and the presence of a narrow neck) and to calculate more accurately the force acting on the ions in the pore. For example, a comparison between the potentials calculated using the 1-dimensional approximation (8) and numerical integration for a cylindrical channel of radius $r=4 \AA$ and length $l=30 \AA$ are shown in Fig. 2(b). It can be seen from the figure that, although (8) provides quite a good approximation for the potential in the pore, numerical integration give a more realistic picture especially at the pore mouth. The cylindrical approximation for the solution of the Poisson equation can be further extended to calculate boundary conditions for the solution at the mouth of the pore using PNP theory in the bulk.

One of the main advantages of using this approximation is that it allows to include qualitatively new physical features into the analysis of the ion-wall interaction while still using a low-dimensional model.

\section{INTERACTION WITH VIBRATIONAL MODES}

As we have mentioned in the introduction one of the main contributions to the selectivity and conductivity properties of the channel comes from the ion-wall interaction. The forces and pressures involved are very large as indicated by estimations based on a mean spherical approximation. ${ }^{4}$ The importance of the dynamical flexibility of the channel has also been investigated. ${ }^{25}$ We would like to comment in this context that the dynamical flexibility of the channel walls may modify exponentially strongly the permeability and the selectivity of the channel if the potential barriers influencing the ion moving through the channel can be modulated, 
either periodically or stochastically. In particular, it is know to have dramatic effect on rate processes in biomolecules ${ }^{10-12}$ and on molecular transport. ${ }^{26,27}$

To include the effect of ion interaction with the walls into the model within the framework of the selfconsistent approach outlined above, one may allow a portion of the wall in the form of cylindrical shell (for example an element of finite volume shown in the Fig. 2(a)) to move in the radial direction, while keeping the cylindrical symmetry of the problem. This will correspond to a uniform compression and expansion of the finite volume element. If we assume that the flexible element has constant mass $M$ and the change of its potential energy due to the extension is $\delta U=\frac{1}{2} k \delta r$, where $\delta r$ is the change of the radius, then the equation for the damped radial motion of the element reads

$$
M \delta \ddot{r}+M \Gamma \delta \dot{r}+k \delta r=F+\sqrt{2 M \Gamma k_{B} T} \nu(t) .
$$

Here we have included damping term $M \Gamma \delta \dot{r}$ and the corresponding random force $\sqrt{2 M \Gamma k_{B} T} \nu(t)$, whose amplitude is related to the damping constant via the fluctuation-dissipation theorem. The ion-wall interaction is modelled by the Coulomb force $F=q_{1} q_{2} / 4 \pi \epsilon \epsilon_{0} R^{2}$, where $R=\sqrt{z^{2}+(r+\delta r)^{2}}$ is the distance from the ion to the flexible element, $q_{1}$ is the total surface charge of the flexible ring and $q_{2}$ is the charge of ion. Eq. (11) describes small oscillations of $\delta r$ that may model the first eigenmode of the vibrations of the cylindrical wall that preserve cylindrical symmetry. The variations of the potential well come from the variation of the distance $R$. Although these variations are very small they may induce exponentially strong modulation of the transition rate. $^{28}$

Eq. (11) must now be solved simultaneously with Eq. (1) where the electrical potential $\phi$ is calculated numerically and tabulated for every combination of the positions of the ion and of the ring (cf. ${ }^{16}$ ). This system of equations allows one to include into the model new important mechanism of the dissipation of the energy of ions passing through the channel. Perhaps more importantly, the model (1), (10), and (11) describes the ion-wall interaction in a self-consistent way, i.e. the ion passing through the channel may induce oscillations of the wall that modulate exponentially strongly the rate of passage of other ions through the channel. This effect may oppose the effect of stabilization of ion in the channel due to the dynamical pore relaxation self-induced by the presence of ion in the pore. ${ }^{25}$ In particular, if one could find conditions under which the ionic motion through the channel is only possible in the presence of the oscillations and is blocked otherwise, these result will bear on the coupling of $\mathrm{BD}$ simulations with one of the possible mechanisms of gating.

\section{CONCLUSIONS}

A novel low-dimensional model of coupling of ions motion to the vibrational modes of the pore is introduced. The model is based on a self-consistent approach to description of Langevin dynamics of ions in open ionic channels. It allows one to include in the simulations a new mechanism of energy dissipation and self-induced exponentially strong modulation of the transition rate of ions through the channel.

\section{ACKNOWLEDGMENTS}

The work was supported by the Engineering and Physical Sciences Research Council (UK), RFBR (The Russian Foundation for Basic Research), INTAS, and ESF.

\section{REFERENCES}

1. B. Hille, Ionic Channel Of Excitable Membranes, Sinauer Associates, Sunderland, MA, 1992.

2. D. Chen, J. Lear, and B. Eisenberg, "Permeation through an open channel: Poisson-Nernst-Planck theory of a synthetic ionic channel," Biophys. J. 72, pp. 97-116, 1997.

3. P. Fromherz, "Electrical interfacing of nerve cells and semiconductor chips," Chem. Phys. Chem. 3, pp. 276$284,2002$.

4. W. Nonner, L. Cattacuzzeno, and R. S. Eisenberg, "Binding and selectivity in l-type calcium channels: A mean spherical approximation," Biophys. J. 79, pp. 1976-1992, 2000. 
5. B. Corry, T. W. Allen, S. Kuyucak, and S.-H. Chung, "Mechanisms of permeation and selectivity in calcium channels," Biophys. J. 80(1), pp. 195-214, 2001.

6. B. Roux, "Valence selectivity of the gramicidin channel: A molecular dynamics free energy perturbation study," Biophys. J. 71, pp. 3177-3185, 1996.

7. W. Im, and B. Roux, "Ion permeation and selectivity of OmpF porin: a theoretical study based on molecular dynamics, Brownian dynamics, and continuum electrodiffusion theory," J. Mol. Biol. 322, pp. 851-69, 2002.

8. W. Im, and B. Roux, "Ions and counterions in a biological channel: a molecular dynamics simulation of OmpF porin from Escherichia coli in an explicit membrane with $1 \mathrm{M} \mathrm{KCl}$ aqueous salt solution," J. Mol. Biol. 319, pp. 1177-1197, 2002.

9. E. Barkai, R. S. Eisenberg, and Z. Schuss, "Bidirectional shot noise in a singly occupied channel," Phys. Rev. E 54, pp. 1161-1175, 1996.

10. Z. R., "Dynamic disorder - passage through a fluctuating bottleneck," J. Chem. Phys. 97, pp. 3597-3589, 1992.

11. D. J. Bicouta and A. Szabo, "Escape through a bottleneck undergoing non-Markovian fluctuations," J. Chem. Phys. 108, pp. 5491-5497, 1998.

12. N. Eizenberg and J. Klafter, "Molecular motion through correlated fluctuating bottlenecks," Phys. A 249, pp. $424-429,1998$.

13. V. Barcilon, "Ion flow through narrow membrane channels: part I," SIAM J. Appl. Math. 52, pp. 13911404, 1992.

14. D. P. Chen, V. Barcilon, and R. Eisenberg, "Constant fields and constant gradients in open ionic channels," Biophys. J. 61, pp. 1372-1393, 1992.

15. R. S. Eisenberg, M. M. Klosek, and Z. Schuss, "Diffusion as a chemical-reaction - stochastic trajectories between fixed concentrations," J. Chem. Phys. 102, pp. 1767-1780, 1995.

16. G. Moy, B. Corry, S. Kuyucak, and S.-H. Chung, "Tests of continuum theories as models of ion channels. I. Poisson-Boltzmann theory versus Brownian dynamics," Biophys. J. 78(5), pp. 2349-2363, 2000.

17. B. Corry, S. Kuyucak, and S.-H. Chung, "Tests of continuum theories as models of ion channels. II. PoissonPernst-Planck theory versus Brownian dynamics," Biophys. J. 78(5), pp. 2364-2381, 2000.

18. W. Im, S. Seefeld, and B. Roux, "A grand canonical monte carlo-brownian dynamics algorithm for simulating ion channels," Biophys. J. 79(2), pp. 788-801, 2000.

19. B. Corry, M. Hoyles, T. W. Allen, M. Walker, S. Kuyucak, and S. H. Chung, "Reservoir boundaries in Brownian dynamics simulations of ion channels," Biophys. J. 82, pp. 1975-1984, 2002.

20. D. Chen and R. Eisenberg, "Charges, currents, and potentials in ionic channels of one conformation," Biophys. J. 64(5), pp. 1405-1421, 1993.

21. R. S. Eisenberg, "Channels, receptors \& transporters," in Biophysics Textbook On-Line, L. J. DeFelice, ed., Channels, Receptors \& Transporters, ch. Permeation as a Diffusion Process, Biophysical Society, Bethesda, MD http://www.biophysics.org/biophys/society/btol, 2002.

22. B. N. Z. Schuss and R. S. Eisenberg., "Derivation of Poisson and Nernst-Planck equations in a bath and channel from a molecular model," Phys. Rev. E 64, p. 036116, 2001.

23. J. Ferziger and M. Peric, Computational Method for Fluid Dynamics., Springer, Berlin, 1996.

24. http://www.lancs.ac.uk/ kaufmani/psn/.

25. A. B. Mamonov, R. D. Coalson, A. Nitzan, and M. G. Kurnikova, "The role of the dielectric barrier in narrow biological channels: A novel composite approach to modeling single-channel currents," Biophys. J. 84(6), pp. 3646-3661, 2003.

26. R. P., "Brownian motors: noisy transport far from equilibrium," Phys. Rep. 361, pp. 57-265, 2002.

27. R. D. Astumian, "Thermodynamics and kinetics of a Brownian motor," Science 276, pp. 917-922, 1997.

28. D. G. Luchinsky, M. J. Greenall, and P. V. E. McClintock, "Resonant rectification of fluctuations in a Brownian ratchet," Phys. Lett. A 273, pp. 316-321, 2000. 